\title{
The Near-Death Experience of a Nonverbal Person with Congenital Quadriplegia
}

\author{
William J. Serdahely, Ph.D. \\ Montana State University
}

Barbara A. Walker, Ph.D. Eastern Illinois University

\begin{abstract}
This article describes the near-death experience (NDE) a 39year-old man born with severe spastic quadriplegia had when he was two and one-half years old. Nonverbal because of cerebral palsy, the man was able to communicate about his experience after he heard a therapist talking to another person about NDEs. The man experienced a fairly typical NDE and has had seven subsequent out-of-body experiences, the latter hypothesized as temporary relief from his physical pain resulting from muscular spasticity.
\end{abstract}

In the fall of 1987, the senior author interviewed an eight-yearold boy who had a near-death experience (NDE) when he was seven (Serdahely, 1989-90). Because of the paucity of pediatric cases in the NDE literature (Morse, 1983; Morse, Castillo, Venecia, Milstein, and Tyler, 1986; Morse, Conner, and Tyler, 1985), a solicitation for pediatric near-death experiencers (NDErs) was placed in various periodicals. With the exception of one pediatric respondent, this inquiry produced several accounts from adults of their childhood NDEs. One of these retrospective accounts was most unusual, and it is this NDE to which the remainder of this paper is devoted.

Dr. Serdahely is Professor of Health Science at Montana State University, and Dr. Walker is with the Department of Health Studies at Eastern Illinois University. Reprint requests should be addressed to Dr. Serdahely at the Department of Health and Human Development, Montana State University, Bozeman, MT 59717. 
This respondent, whom we shall call Stuart, is 39 years old at the present time, and has been challenged since birth with cerebral palsy. He is able to understand what is said to him at a high receptive level but is unable to speak. However, Stuart can make intelligible sounds for "Yes" and "No," and has gone to great lengths to communicate. His health care providers say he uses his normal intellectual capacity in "unusual [i.e., extraordinary] ways."

He was also born with severe spastic quadriplegia, a painful condition that left him with little mobility and limited physical abilities. His caregivers note his remarkable motivation to overcome his physical challenges. He is able to use a chin switch to operate a computer, thereby allowing him to communicate through the use of preprogrammed messages and "Yes/No" responses as guided by others. Recently, his occupational therapist has devised a unique computer program for Stuart. He can write original sentences with personal messages through the use of adapted software that translates numbers and Bliss symbols into English.

\section{Methodological Limitations}

While Stuart was questioned closely about his NDE through the use of "Yes/No" questions, as with all near-death experiencers we are ultimately left with trusting that the respondent has recalled the experience accurately and transmitted the experience faithfully to the researcher. It is, of course, possible that a childhood NDE could be reinterpreted in light of later events in the childhood, adolescent, or adult years.

While we can never be completely certain of the accuracy of any case, we can take some measure of assurance given the following. First, we know that children who have NDEs report patterns and motifs very similar to those reported by adults recalling their childhood NDEs (Bush, 1983; Morse, Conner, and Tyler, 1985; Serdahely, 1989-90). Second, it is well established that while a particular cultural or religious background may influence the interpretation of an NDE, the basic event or phenomenon seems to remain constant regardless of culture or religion (Ring, 1980). So in the end, we as near-death researchers are left with a subjective impression of the veracity of the respondent, unless obvious inexplicable disparities occur in the respondent's testimony. Such was not the case with Stuart.

Critics of near-death research often ask how we know a person is not making up this experience or just describing something about which 
he or she has read. We know that Stuart's descriptions are not the result of his reading about NDEs because he cannot read. Of course, it is possible that Stuart could have viewed television programs on NDEs, heard radio shows, or listened to someone read aloud about NDEs. However, we asked Stuart if any other these possibilities were the case, and he replied in the negative.

\section{Stuart's NDE}

In the fall of 1988, in Stuart's presence, his occupational therapist was comforting a person who had recently lost a loved one. Audrey, the therapist, had a copy of Raymond Moody's The Light Beyond (1988) and was citing information from the book in an attempt to console that person. She was telling the grieving person about NDEs and how pleasant NDErs report dying seems to be. As Audrey was describing parts of that book, she noticed Stuart had become excited and enthusiastic. Through a series of questions, she ascertained that Stuart had had an NDE when he was two and a half years old, and because of his speech limitations, he had had to wait almost 37 years to communicate about his childhood NDE.

According to information supplied by Stuart's adopted mother, when he was two and a half he was very ill. A precise diagnosis of the illness was never made, but Stuart was said to have had a high fever, increased spinal fluid pressure, and muscular contractions with increased spasticity. It was during that period of illness that Stuart had his NDE. The memories of his NDE remain vivid, even though almost 37 years have elapsed since his near-death experience. Stuart had what Kenneth Ring would classify as a Stage 5 NDE (Ring, 1980).

During his NDE, Stuart felt peaceful and had no pain. He had a outof-body experience (OBE) during which he was able to look down upon his body and the doctors and nurses ministering to him. He did not recognize his body at the time of his OBE. Stuart's NDE did not involve going into a tunnel or a darkness. Instead, he found himself in a misty, bright, and narrow stairway with steps. The end of the stairway was very bright, and there Stuart had a sense of being loved. However, he was quite sure the bright light at the stairway's end was not God.

While he was in the light he did not see trees or lakes, but he did hear "heavenly" music. He also saw about 20 spirits whom he did not know and who did not communicate with him. These spirits were not people close to him (i.e., they were not deceased friends or relatives), but they did make him feel welcomed and loved. He also saw a border around 
the place in which he found himself. The border had a gate that one could pass through. Stuart felt that if one went beyond the border, one would not return to one's body. He could see people on the other side of this border.

Time did not exist in this place. An even brighter light appeared to Stuart, and he knew this light was "God." Communication between "God" and Stuart occurred through thought transmission. "God" told Stuart he had to go back to his body and he did not have a choice about returning, but He did not say why Stuart had to return to Earth. While in this "heavenly" place, Stuart saw the future. He saw himself ablebodied as a boy, as a teenager, and as an adult. (At first, Stuart seemed to be indicating he saw himself in the future on Earth, in perhaps a future incarnation, but after further questioning, it seemed he might be talking about a future life in heaven.)

During the time Stuart was out of his body, he had mobility, and was able to float wherever he wanted to go. He remembers seeing and being able to move his hands and arms, but not his legs and feet.

Stuart has had two main aftereffects of his NDE, which relate well to Ring's (1984) findings on aftereffects of NDEs. First, Stuart has no fear of death. As a result of his NDE, he believes there is no such thing as death and that we continue on as "spirit" persons. The second aftereffect is a series of out-of-body experiences subsequent to his NDE. Stuart has indicated that he had four OBEs before he was 21 years old and three OBEs after that age.

During some of his OBEs, he would float up past the ceiling, over the metropolitan community in which he lives, and out into the sky. Sometimes he was met by Jesus, who was nurturing and loving to Stuart, and who embraced, held, and comforted him. It was during these times that Stuart was told he had two purposes for his life, one of which is that he has a message for people with disabilities and things to teach them.

In February 1989 Stuart had his sixth out-of-body experience. He indicated he went to "Paradise" while asleep and saw a glowing woman with a Scandinavian accent who had died a century ago. Stuart believed she was an ancestor of his, and he was thrilled at how loving she was. He also saw two deceased grandfathers who touched him, two deceased grandmothers, and two deceased stepbrothers. (His adopted mother confirmed that she had lost two sons prior to Stuart's OBE.) $\mathrm{He}$ also saw others there whose genders he could not determine, and indicated it was like a "big reunion." He also indicated he talked with God and Jesus, who encouraged him to write his autobiography to help others with handicaps. 


\section{Discussion}

Two lessons emerge from Stuart's near-death experience and its concomitants. One lesson has to do with our bodies and our body images, and the other pertains to pain and being pain-free.

Stuart's body is immobile and severely crippled because of quadriplegia. Just to communicate about basic needs, he has to use his chin switch to activate his computer program. And yet when he had his outof-body experiences, including the one associated with his NDE, he was quite mobile and able to move his arms and hands freely and easily. It is interesting to note that during his OBE when he nearly died as an infant, he, like some other NDErs, did not recognize his body (Ritchie, 1978).

Phyllis Atwater (1988), in writing about her three NDEs, made the point that we are not our bodies. She likened the body to a jacket, which is discarded when worn out, an analogy made by Eknath Easwaran (1978) and other authors. Atwater believes, just as Stuart does, that the spirit body survives and continues after the physical body dies and deteriorates.

Stuart believes he was sent back to his infirm body with the mission of imparting a message to others with disabilities. But he also has a message for those who are able-bodied, a message that seems most timely and appropriate for a culture that so highly values youthfulness and promotes and sells so many products to "improve" our physical appearance.

It is our belief that Stuart's message to able-bodied persons is that our bodies are an illusion and that who we are, just as who Stuart is, is far greater than the physical body. It is the consciousness or spirit, the energy that separates from the body during an OBE, that according to Stuart and other NDErs (Grey, 1985), never dies. Another of the senior author's respondents, who has had approximately $50 \mathrm{OBEs}$, stated that the consciousness that separates from the body, compared to the body, is like the physical body compared to a toenail clipping.

Stuart, residing in a body so malformed, also has a corollary message for the able-bodied. That second message is nicely summarized in the psychological evaluation done on Stuart in August 1988, which reads, "Attempts should be made to desensitize peers to Stuart's physical handicaps, and have them made known of what a wonderful and determined individual he is." In other words, for us nondisabled persons, the lesson is to look beyond the physical disabilities that can distance and separate us and to see the many things that can bring us together, the many things that other NDErs claim unite us, such as 
our capacity to help others and to love one another unconditionally (Moody, 1988; Flynn, 1986).

The second major lesson of Stuart's story relates to pain and pain relief. Stuart is frequently in a great deal of pain. At times during our conference telephone calls we would have to stop because he was in such great physical discomfort. We know that Stuart believes he has had at least seven out-of-body experiences since his NDE. These OBEs may provide some relief, albeit temporary, from the almost constant pain he experiences while he is in his body.

The pain relief Stuart received from his OBEs is not unlike the relief felt during the NDEs or OBEs of victims of sexual abuse or physical abuse (Serdahely, 1987-88). These victims report the OBE is a way of dealing with something quite painful and traumatic, and leaving the physical body temporarily relieves their terrible emotional and physical pain. NDErs have consistently reported that there seems to be no pain on "the other side" (Moody, 1975). However, Stuart cautions that one is not to search for these experiences to relieve pain; these experiences are given as spontaneous gifts.

\section{References}

Atwater, P. M. H. (1988). Coming back to life. New York, NY: Dodd, Mead.

Bush, N. E. (1983). The near-death experience in children: Shades of the prison-house reopening. Anabiosis: The Journal of Near-Death Studies, 3, 177-193.

Easwaran, E. (1978). Meditation: Commensense directions for an uncommon life. Petaluma, CA: Nilgiri.

Flynn, C. P. (1986). After the beyond: Human transformation and the near-death experience. Englewood Cliffs, NJ: Prentice-Hall.

Grey, M. (1985). Return from death: An exploration of the near-death experience. London, England: Arkana.

Moody, R. A., Jr. (1975). Life after life. Covington, GA: Mockingbird.

Moody, R. A., Jr. (1988). The light beyond. New York, NY: Bantam.

Morse, M. (1983). A near-death experience in a 7-year-old child. American Journal of Diseases of Children, 137, 959-961.

Morse, M., Castillo, P., Venecia, D., Milstein, J., and Tyler, D. (1986). Childhood neardeath experiences. American Journal of Diseases of Children, 140, 1110-1114.

Morse, M., Connor, D., and Tyler, D. (1985). Near-death experiences in a pediatric population. American Journal of Diseases of Children, 139, 595-600.

Ring, K. (1980). Life at death: A scientific investigation of the near-death experience. New York, NY: Coward, McCann and Geoghegan.

Ring, K. (1984). Heading toward omega: In search of the meaning of the near-death experience. New York, NY: William Morrow.

Ritchie, G. (1978). Return from tomorrow. Waco, TX: Chosen Books.

Serdahely, W. (1987-88). The near-death experience: Is the presence always the higher self? Omega, 18, 129-134.

Serdahely, W. (1989-90). A pediatric near-death experience: Tunnel variants. Omega, $20,55-62$. 